\title{
Blockchain, Artificial Intelligence, Internet of Things to Improve Governance, Financial Management and Control of Crisis: Case Study COVID-19
}

https://doi.org/10.21272/sec.4(2).78-89.2020

Belen Suarez Lopez, ORCID: https://orcid.org/0000-0002-6323-2621

Reacción Economica, Lead Economist Researcher and CEO; ISO56008 Member Expert for Innovation Measurements at ISO - International Organization for Standardization, Spain

Antonio Vargas Alcaide, ORCID: https://orcid.org/0000-0002-9791-2377

Economist Research, Project Manager Professional at Blockhub, Spain

\begin{abstract}
Today, the coronavirus infection COVID-2019 deals a devastating blow to the economies of most countries due to disruption of production chains, bankruptcy of small and medium-sized businesses, increasing the number of unemployed and more. Under these conditions, the coverage of digitalization of all sectors of the economy and basic spheres of life of citizens becomes especially important. The article is devoted to the analysis of the possibilities of the latest blockchain technologies, artificial intelligence and the Internet of Things in view of their impact on the transformation of the business process management system. The study used methods of bibliographic analysis of scientific publications and analytical reports of international organizations related to the concept of "Industry 4.0" and diseases of coronavirus infection, analyzing from the audit point of view, how to reinforce the principles of transparency, responsibility, and participation. It has been proven that blockchain technology is able to service online payments without intermediaries, receipt and transfer of digital assets, as well as political elections and voting. Artificial intelligence models can help map, manage, predict, and model complex processes, reducing uncertainty, and supporting professionals in decision-making. The Internet of Things allows you to transfer information, improve control and automation, and provides opportunities to optimize the company's operating costs. The result of the study can be practically valuable for many stakeholders: auditors - conducting audits by artificial intelligence; public administration developing measures to address the economic, social and political crisis triggered by the pandemic, by building trust between government and citizens through communication, and by ensuring transparency and accountability.
\end{abstract}

Keywords: blockchain, artificial intelligence, Internet of Things, COVID-19, health care crisis, economic crisis, political crisis, control, public administration, financial management.

JEL Classification: H12, H83, I15, M48, O35.

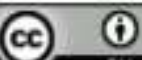

Cite as: Lopez, B.S., Alcaide, A.V. (2020). Blockchain, AI and IoT to Improve Governance, Financial Management and Control of Crisis: Case Study COVID-19. SocioEconomic Challenges, 4(2), 78-89. https://doi.org/10.21272/sec.4(2).78-89.2020

(C) The Authors, 2020. This article is published with open access at Sumy State University.

\section{Introduction}

This article aims to analyze whether new technologies can provide tools to improve governance, risk management, and control processes. The study is based on a bibliographic review of scientific sources of the main characteristics of technologies, the main challenges of the COVID-19, and the main approaches to solving the problem.

Many countries around the world face unprecedented challenges due to COVID-19, the pressure on governments is extreme, and the impact on people continues to grow. Governments, civil society, and private corporations are looking for ways to help. Governments should lead coordination from a strategic approach that generating and sharing value with the collaboration of the private sector, public administrations, and the academic world, adding 
focus to a Quadruple Helix for citizen integration. In times of disaster response and relief, good governance values can come under intense pressure, but they can also contribute significantly to better results.

Other factors have been added to the characteristics and severity of the virus, such as the insufficient structure of health systems, the lack of coordination and control in management between governments and administrations, the poor investment in research of the last decade and the low resilience of some productive sectors in a confined situation. At the beginning of the industry 4.0 era, emerging technologies such as Blockchain, AI, and IoT might be able to offer solutions for managing global pandemics in the medium term. However, face to the harshness of the consequences that isolation, a strategy most commonly accepted as a short-term solution by governments glimpses a new economic and social order whose profile will be conditioned by the characteristics of the emergencies from which it arises. These are the health emergency, economic emergency, social emergency, and political emergency.

Develop digital platforms or applications to keep citizens informed, allow public participation, and/or offer open data; and design and implement digital tools to allow public participation. The development of digital and/or crowdsourcing provision of public and government services and the fight against disinformation off/online. But at the same time, governments must strike a balance with data rights protection and privacy. All of them, urgent issues to resolve that highlight the need to improve Governance, Risk Management, and Control Processes in a necessarily digitized world.

\section{Literature review}

This paper summarizes the arguments and counterarguments within the scientific discussion on the issue. The scientific literature has been complemented with articles and research from technology developers, audit experts, and the dissemination provided by international institutions reports to provide a big picture and integrate the main stakeholder's point of view, taking into account that the technologic applications are in an initial stage, as well as, given the high degree of uncertainty associated with the pandemic, being still too early to anticipate the possible epidemiological evolution on the part of the scientific community, and as a result, the difficulty of anticipating the impacts generated by the emergency, both economically, financially, socially and politically.

The scientific literature has been complemented with articles and research from technology developers, audit experts, and the dissemination provided by international institutions reports.

The literature review can be classified into three main blocks:

1. The first block, definition, and main features of the technologies providing the point of view both academics, for example, Suarez et al. (2019) about Blockchain or Lopez (2013) related to IoT, and developers about of value proposition of these technologies, as Narula et al. (2018) and anticipate its potential to assess the socio-economic impact, EU Blockchain Observatory, and Forum (2020).

2. The second block, analysis of applications in the field of auditing, based on the scientific review, such as Dai and Vasarhelyi (2017) or Cheong et al. (2020), and the reports of the expert auditors regarding the potential of these technologies to improve Governance, Financial Management and Control Processes, ACCA (2019).

3. The third block, Situational Judgement regarding the COVID-19 pandemic from the understanding of the problem root causes to potential solutions summary from the academic perspective, as it is proposed by Micali (2020) and the reports of international institutions, for example, Colombo (2020).

\section{Methodology research and methods}

Since a scientific literature review is made during the initial state of the pandemic, the high level of uncertainty and the most of the technology applications are in development, This scientific research has been based on a qualitative analysis, which should be confirmed by subsequent empirical analysis.

The research is developed from the main hypothesis: The set of technologies under the Blockchain, AI, and IoT umbrella could potentially improve Governance, Financial Management and Control Processes, and as a consequence, enabling the development of technological applications and innovation tools to overcome crises such as the Covid19 pandemic. 
The research is a bibliographic review of scientific literature and reports from international institutions. The research begins from the conclusions reached by the preliminary and complementary research of the authors in the article Blockchain Facing Socioeconomic Challenges, Promise Versus Probability (Suarez et al., 2019).

The developed methodology starts from the individualized analysis of new technologies such as Blockchain, AI and IoT, to understand what their strengths and weaknesses are to build viable and effective solutions towards the improvement of governance, financial management, and control conceptualized within the COVID-19 crisis.

In a second stage, the research analyzes the COVID-19 crisis in the three main associated crises, the health crisis, the economic crisis, and the political crisis, working from the evaluation of the root causes of the COVID-19 crisis and its challenges fundamental to assess how could promote these technologies feasible and practical solutions to overcome the crisis and its impacts.

The research approaches the study from the auditor's view, as representative of the necessary critical spirit with which citizens must evaluate the actions of institutions and governments when analyzing governance processes, control systems and the use of financial and non-financial resources.

\section{Results}

Given the complexity and global dimension of the problem at the ecosystem level, it is necessary to build solutions from a strategic point of view, due it is a problem that affects the whole world ecosystem, the extremely high degree of uncertainty in the evolution of the pandemic and the devastating consequences on social, and economic systems.

Strategic thinking and scenario planning, often used in military situations, enable decision-makers and institutions to take new heights through differentiated vision, rigorous prioritization, intentional resource allocations, and increased morale through confidence in leadership (Friga, 2020). The management of the COVID-19 crisis has revealed the need for planning, organization, direction, and control (Escola and Kadi, 2017) not only to overcome the health crisis but also to face the impact caused in the economy, society, and politic systems.

Among the benefits of deciding from strategic thinking, we highlight decision-making in light of future consequences, develop a coherent and defensible basis for decision-making, improve organizational legitimacy, enhance performance and efficiency, increase the responsiveness and resilience and last but not least, they effectively address rapidly changing circumstances (Mawela, 2012).

Identifying the drivers for change is a crucial step in the processes of strategic thinking, so this research begins to study the main characteristics of the new technology to understand the potential of face the socio-economic challenges caused by COVID-19 and improve governance, financial management, and control of the crisis.

\subsection{Blockchain}

Blockchain is just a digital record, a digital event that is distributed or shared among many different parts within an ecosystem (nodes), so it is distributed. It can only be updated based on the consensus of the majority of the participants in the system, and once the information has been entered, it cannot be deleted (or, at least, easily deleted). It is therefore immutable. The ledger is often secured through an intelligent combination of cryptography and game theory and does not require reliable nodes such as traditional networks. It can be verified by third parties, so it is reliable. The records may contain different types of shared data: transaction records, document attributes, credentials or other data. This information is stored chronologically in chained blocks, so each chain contains an accurate and verifiable record of the modifications that have been made in its history (Suarez et al., 2019).

The added value of Blockchain is that it greatly enhances performance in areas where inefficiencies are introduced by the so-called 'efficiency visibility' and 'trust 'deficits, as consequence, it could represent an important tool for help to improve governance and financial management and control both in the private and public sector.

For Auditors, distributed ledgers become a sort of universal bookkeeping service, removing the need to reconcile multiple databases of records and providing a perfect audit trail. Blockchain offers the possibility of generating exception reports that are based on all transactions rather than on using sampling techniques. Today's audit cycles could potentially be replaced by more frequent or even continuous, real-time, audit. The removal of mundane tasks will bring the contribution of the auditor's judgment into stronger focus. (ACCA, 2019).

One important area into the blockchain scope is the use of smart contract, it is a piece of code that, once stored on the blockchain, does their task autonomously (Suarez et al., 2019). Blockchain's functions of data integrity 
protection, instant information sharing, and programmable and automatic controls of processes via smart contracts could facilitate the development of a new accounting ecosystem and enable a certain level of automatic assurance. (Cheong et al.,2020). As businesses are participating increasingly in transactions involving cryptocurrencies, "it is becoming common for financial statements to show material cryptocurrency balances and to reflect the results of cryptocurrency transactions" (CPA Canada, 2018).

Distributed ledgers as blockchain enable financial institutions to efficiently reconcile cross-organization transactions. For example, banks might use a distributed ledger as a settlement log for digital assets. Unfortunately, these ledgers are either entirely public to all participants, revealing sensitive strategy and trading information, or are private but do not support third-party auditing without revealing the contents of transactions to the auditor. Auditing and financial oversight are critical to proving institutions are complying with regulations. (Narula, et al.,2018).

Distributed ledgers enable real-time validation by all participants (known as public verifiability), but at the cost of privacy (every participant must download all transactions to verify their integrity). This is untenable for institutions that rely on secrecy to protect strategy and intellectual property (e.g. trading strategies), and for organizations that have to comply with laws and regulations around data privacy, for example, the General Data Protection Regulation in Europe.

Distributed ledgers that support privacy generally operate in one of two ways: either by only committing to hashes of transactions on the ledger using trusted third parties to independently verify transactions or by using cryptographic commitment schemes to hide the content of transactions.

For digital books could be elements of control systems and to allow the auditing of transactions, they should overcome three key challenges:

1. Provide privacy and audit. Preserve the privacy and at the same time allow an auditor to calculate likely correct measurements of the data in the ledger. ZkLedger proposes a system combining several primitive cryptographic. Through the use of Pedersen commitments, allowing a verifier to, for example, add up the inputs, conserving assets. Besides, the auditor may combine commitments to calculate linear combinations of values in different rows in the general ledger.

2. Integrity, making it easier to complete the audit while maintaining privacy, making sure that during the audit transactions to hide assets are not omitted, despite not disclosing their actor's identities.

3. Efficiency, they implement a series of optimizations, generating assets and audit tests, these are the consistency tests and the audit tokens, both of which are publicly verifiable, non-interactive and are encoded for each identity.

The next figure represents the overall zkLedger system, design showing the interactions between the three main entities (banks, auditor, and the shared ledger) into the system. Each bank maintains a private state, consisting of the transaction database for transactions the bank originated, and the bank's secret key.

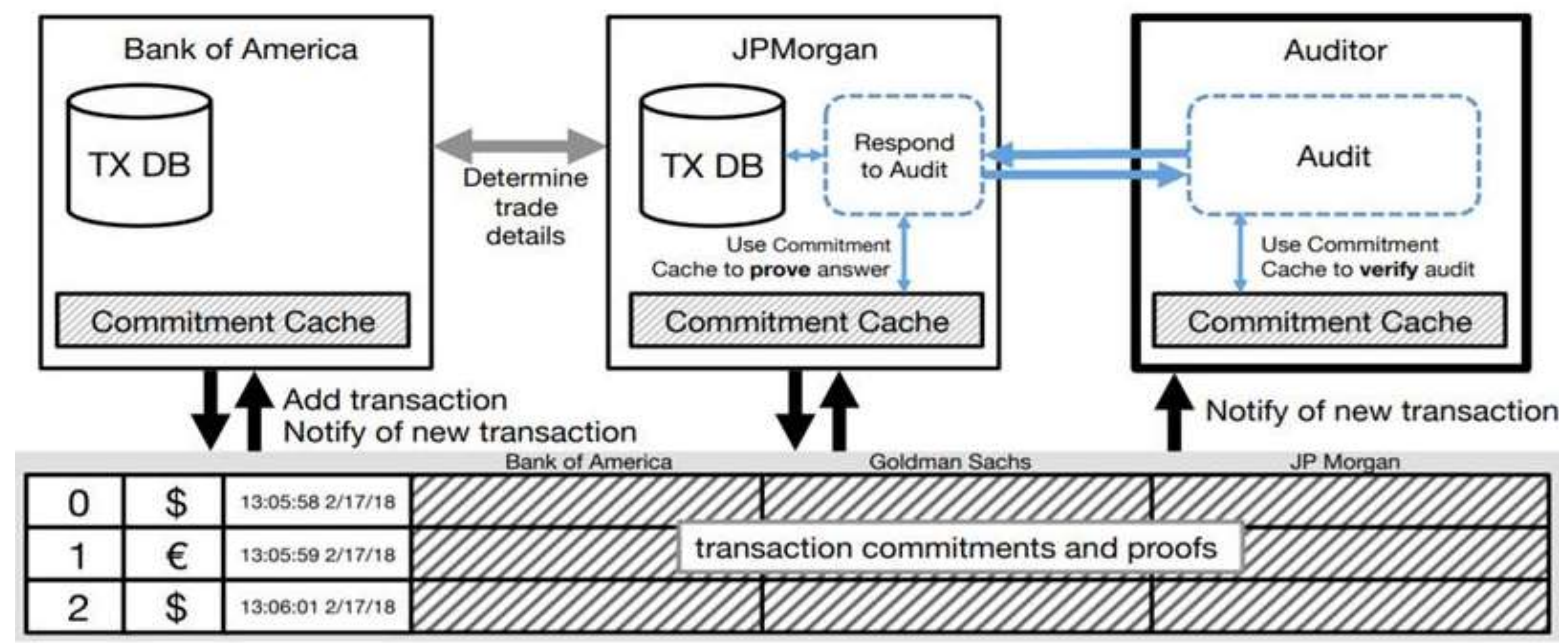

Figure 1. Overall zkLedger system design showing the interactions between three main entities (banks, auditor and the shared ledger) in the system

Notes: Each bank maintains private state, consisting of the transaction database for transactions the bank originated, and the bank's secret key. 
In this way a distributed accounting system is established that combines strong privacy and complete auditing, combining primitive fast cryptography using audit tokens and allowing to map/reduce to probably calculate the correct answers to the auditor's queries. (Narula et al., 2018).

\subsection{Artificial intelligence}

It is sometimes difficult to establish a specific definition for this discipline, so AI can mean different things to different people. It might be more accurate to understand AI as a general term for a group of technologies that can be combined in different ways. For some, AI is about artificial life forms that can surpass human intelligence, and for others, almost any data processing technology can be called AI. A more useful approach would be to consider the properties that are characteristic of AI, autonomy, and adaptability. Autonomy could be defined as the ability to perform tasks in complex environments without the constant guidance of a user. Adaptability, meanwhile, would be the ability to improve performance by learning from experience.

AI is a scientific discipline, therefore, it represents a collection of concepts, problems and methods to solve them. There are concepts related to AI between which it is necessary to discern, despite being closely related, to understand its full potential. These include machine learning, data science, and deep learning.

Machine learning (ML). Systems that improve their performance in a given task with more and more experience or data. Machine learning can be said to be a subfield of artificial intelligence, which is itself a subfield of computing. Machine learning enables AI solutions that are adaptive. An example would be the use of ML in risk assessment, "supervised learning algorithms can be used to help identify specific types of characteristics that warrant greater scrutiny, and improve targeting areas of focus for the audit" (ACCA, 2019).

Deep learning (DL) is a subfield of machine learning, the "depth" of deep learning refers to the complexity of a mathematical model, and that the increased computing power of modern computers has allowed researchers to increase this complexity to levels that do not appear only quantitatively but also qualitatively different from before.

The interrelation between theses fields could be outlined as follows:

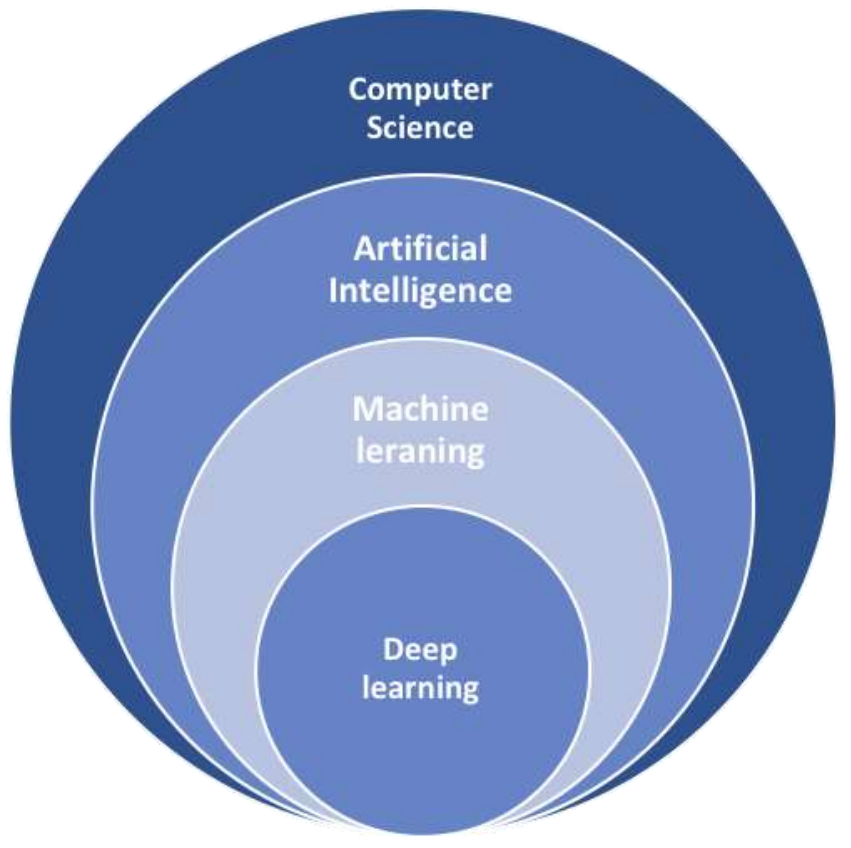

Figure 2. Interrelation graph between AI disciplines

Source: own elaboration source.

Data science is a recent general term (a term covering several sub-disciplines) that includes machine learning and statistics, certain aspects of computing that include algorithms, data storage, and web application development. Data science is also a practical discipline that requires an understanding of the domain in which it applies, for example, in business or science: its purpose (meaning "value-added"), basic assumptions, and constraints. Data science solutions often involve at least a pinch of artificial intelligence. Between the potential 
applications, as an example could highlight the use on Audit, where Data analytics allow auditors to use $100 \%$ of a population's transactions when performing their tests.

Robotics means building and programming robots so that they can operate in complex, real-world scenarios. In a way, robotics is the ultimate challenge of AI since it requires a combination of virtually all areas of AI. Within the potential application, we could speak about computer vision and speech recognition for sensing the environment. Many of the robotics-related AI problems are best approached by machine learning, which makes machine learning a central branch of AI for robotics.

Between the weaknesses and risks that must be overcome related to AI, algorithmic bias and lack of transparency and the existence of barriers to entry should be highlighted.

Algorithmic Bias. AI, and in particular machine learning, is being used to make important decisions in many sectors, making it necessary to address an important concept - the concept of algorithmic bias or a tendency to discriminate based on ethnic origin, gender or other factors, such as when making decisions about job applications, bank loans, etc.

The main reason for algorithmic bias is human bias in the data and represents a real phenomenon that currently affects a multitude of people daily. AI systems are only as good as the data that are introduced into them. Bad data can contain implicit racial, gender, or ideological biases. Many AI systems will continue to be trained using bad data, making this an ongoing problem. Some scientific in the field believe that bias can be tamed and that the AI systems that will tackle bias will be the most successful.

A crucial principle, for both humans and machines, is to avoid bias and therefore prevent discrimination. Bias in the AI systems mainly occurs in the data or the algorithmic model. As we work to develop AI systems we can trust, it's critical to develop and train these systems with data that is unbiased and to develop algorithms that can be easily explained.

The lack of transparency that could be overcome from the regulation. An important step towards transparency is the European General Data Protection Regulation. It requires that all companies that reside within the European Union or that have European customers must:

Upon request, disclose what data they have collected about anyone (right of access)

Delete any data that is not obliged to fulfil other obligations when requested (right to be forgotten)

Explaining the data processing performed on the customer data (right to explanation)

However, it is still unclear what exactly counts as an explanation. For example, iwhat about deep neural networks that easily involve millions of parameters trained using terabytes of data? The discussion on technical implementation about the explainability of decisions based on machine learning is currently intense.

Barriers to Entry. Another challenge to face for AI to deploy its full potential is the concentration in the sector due to the barriers to entry generated by the cost of collecting, storing, and processing these large data sets coupled with the cost of hiring AI experts, making its development acceptable only by large companies that concentrate access to technology.

Some of these challenges could be mitigated by combining AI with Blockchain applications in different ways, for example, blockchains can be used, to develop open, decentralized data markets in which data producers, be they individuals or companies, could sell, rent, or share their data (EU Blockchain Observatory and Forum, 2020).

In the Audit scope, applying AI and ML algorithms could improve the quality of analysis and the forecasts, and at the same time, increase the fraud detection rate. As it is necessary to combine internally generated data in large quantities with external data from a diversity of information sources, it becomes more complex to manage and analyze it, tools with DL increases the range of data that can be handled and increase the capacity of analyzing data through and outside corporate data silos enhancing organizations' ability to detect opportunities, avoid threats, make better decisions while enabling a more democratized process across the organization (ACCA, 2019). 


\subsection{Internet of Things (IoT)}

IoT describes a system where the elements in the physical world and the sensors within or connected to these elements, which are connected to the Internet through wired and wireless Internet connections (Lopez, 2013). These devices are intended to gather and disseminate data autonomously and, increasingly, to act autonomously in this data to perform tasks. In a broad IoT sense, all types would be included connected sensors and meters, actuators (devices that do something in the physical world, like robots or drones), as well as the software that runs them (EU Blockchain Observatory and Forum, 2020).

There are multiple applications of IoT, and within the strengths of this technology we can refer to "The three Cs of IoT" (López, 2013):

Communication. IoT communicates information to people and systems, such as the health and condition of the equipment (for example, on or off, charged, full or empty) and data from sensors that can monitor vital signs. In most cases, this is information that we did not have access to or that was collected manually very infrequently. Therefore, GPS-enabled assets can communicate your current location and movement. Location is important for moving items, such as in healthcare, for example, IoT can help a hospital track the location of everything from wheelchairs to cardiac defibrillators and surgeons. In the transportation industry, a company can offer real-time tracking and status of packages and pallets. For example, you can use sensors to track the location of a refrigerated shipping container and its current temperature.

Control and Automation. In a connected world, IoT makes it easy to see the condition of a device by allowing multiple functions, such as remotely turning on or off specific equipment, adjusting the temperature in a climate-controlled environment, unlocking a car, or turning on the washing machine. Unmanned drones are used in a variety of commercial projects, such as energy line inspection and its potential for use in inventory inspection, particularly where physically scale or distribution is a problem Drones are the aerial component of the Internet of Things, the constant growth in the number of devices and sensors connected through IP (Internet Protocol). This technology brings great advantages to sectors such as auditing and assurance in areas such as agriculture (ACCA, 2019).

Cost savings. Once a performance baseline has been established, a process can send anomaly alerts and possibly deliver an automated response. The measurement provides actual equipment health and performance data, rather than just estimates. With new sensor information, IoT can help save money by minimizing equipment failure and allowing planned maintenance to take place, and there are also important applications to save energy consumption, providing data so that users can understand the process. Energy consumption and analyzing opportunities to save costs, it is the case of sensors that analyze a vehicle's driving behaviour and speed, to reduce fuel consumption or tire wear.

Applying to the scope of COVID-19, The traceability of people positive cases of coronavirus and their social contacts both in the incubation period and the development of the disease, as well as the traceability of resources, the use and forecast of medical equipment, monitoring in the supplier value chain, the administration of epidemiological data that needs to be collected massively on IoT devices and that, can be shared and analyzed as a group

On the side of IoT weakness, there are numerous challenges to overcome such as:

Security. The ability to monitor and control millions (if not billions) of heterogeneous devices, to help them communicate and transact with each other while keeping them secure. IoT devices, for example, come together with data from the environment through sensors and can be used to interact directly and autonomously with it. But they can also be susceptible to piracy and, to be useful, they need a means of transmitting and receiving information securely and interacting with each other.

Scalability. As IoT continues to grow around centralized solutions, it brings associated bottleneck problems when the associated devices grow, so we can see how some solutions in use today are reaching their limits.

Interoperability between IoT devices, making data transfer between different devices or platforms difficult. The development of convergent platforms, based on Blockchain and combined with AI and/or IoT, could largely solve these limitations and allow them to develop their full potential, starting from decentralized solutions in Blockchain, such as platforms where devices pack data and share them with the decentralized 
platform offering a more scalable, more robust and more direct approach. (EU Blockchain Observatory and Forum, 2020).

After analyzing the main attributes of the technologies, we will now turn to study the situation generated worldwide by the Covid-19 pandemic.

\subsection{Covid_19 case study}

Many countries around the world are facing unprecedented challenges from COVID-19 the strain on the governments is extreme, and the impact on people all over the world continues to grow. Between the social initiatives, the activism is participating intensively, so we can see actions such as the citizen-led community responses, including neighbourhood volunteer groups and neighbourhood associations, clergy, teachers, or others helping to inform the public on the risks and needed steps, however, the institutions need to lead participatory disaster response strategies, including working with civil society and citizens.

4.4.1. Health emergency. The unprecedented health emergency in recent decades is caused by the interaction of variable variables, first the characteristics of the virus, second the lack of resilience of health systems to face the disease and thirdly the difficulty of monitoring at the epidemiological level as a consequence of the scarcity and lack of harmonization in data management for monitoring and control.

The most effective means to stop the pandemic is the massive deployment of an effective vaccine, however, given the lack of an effective vaccine for the entire population in the short term, other tools to combat infectious diseases for vaccines are available such as identifying transmission chains and breaking them by isolation or treatment (Micali, 2020).

Innovative digital solutions are also emerging. Access to telemedicine has been facilitated in France and the United States. Israel has introduced robotic devices and the use of telemedicine to monitor the health status of people in quarantine. Korea is testing smartphone applications to allow quarantined people to report on the progress of their case, as well as to monitor their quarantine compliance. Artificial intelligence initiatives to track the spread of the virus and predict where it might appear next have been developed in Canada. Several apps to tracing contact are in development (Colombo, 2020).

Health systems policy responses can be organized along with three key S priorities: mobilizing staff to diagnose and treat patients; supplies of the equipment necessary to safely diagnose people and provide them with treatment acute when needed; and space to diagnose people quickly and safely, to isolate confirmed and suspected cases, and to treat patients in the hospital or at home (Scarpetta, 2020).

Undoubtedly, the mapping of the cases, as well as a multivariable regression analysis would facilitate the promotion, at least numerically, of the creation of policies in the fight against the pandemic and the measurement of their impacts, whether negative or positive, for example, the reduction of fine particle emissions, factor to consider, according to the studies that determine the correlation between virus transmission and environmental contamination (Setti, 2020). This kind of analysis could be deployed by data provided by smart devices of IoT, stored in blockchain platforms, and analyzed by AI models.

In the case of COVID19 tracing apps, homomorphic encryptions of blockchain platforms would allow governments, say, to supply telecoms companies with lists of infected patients and ask for their recent whereabouts as well as the identities of people they've met - all in a limited anonymous way, respecting privacy (Micali, 2020).

4.4.2. Economic crisis. The rapid growth in the number of people infected with COVID-19, the disease caused by the new SARS-CoV-2 coronavirus, is not only overloading health systems, causing large-scale loss of life and severe human suffering, but it presents a great threat to the global economy, specifically affecting the most vulnerable and further spreading a social fabric challenged by high levels of inequality (Colombo, 2020).

The current uncertainty is very high, however, looks clear that the second great economic and financial crisis of the 21 st century is emerging. Economic indicators indicate that the magnitude of the recession will be historic with quarter-on-quarter falls never seen before caused by the measures of confinement, and restriction on the mobility of the countries, generating a shock of simultaneous supply and demand and global level.

Measures are requested from all economic sectors, economic stimulus measures that could prevent the shock from ending up having persistent structural effects in the economy, among these measures are the forms of 
liquidity support, for families, the self-employed and companies, to allow adjusting the activity of companies and, at the same time, maintaining employment seeking to maintain the link between workers and companies.

However, the magnitude, breadth, and speed of the stimulus packages of governments and institutions, make the financing needs of the public sector increase abruptly, forcing Central Banks around the world to be proactive so that the sustainability of public debt is not questioned, with the implementation of massive asset purchase programs to give coverage to the increase in financing needs of the public sector and avoid the development of the second great Financial Crisis of the 21st century.

The magnitude of the economic impact will depend on the time in which they have to maintain active measures of confinement, and social distancing, reduction of mobility, and economic activity according to the evolution of the pandemic.

The challenge is that fiscal support measures are not up to the economic impact of the pandemic. However, if the management is adequate, to overcome the threats and turn the crisis into a source of opportunities, reinforcing the health systems, redesigning the production model at a global level, relocating production from the manufacture centres to the sale points, redefining strategic sectors, deploying new models of the circular economy, enabling new ways of working, and creating a more humane, smart, sustainable and environmentally friendly economy.

Most of the measures that are being considered continue to exclude a very important percentage of the population, both in access to health systems and to the proposed tax aid. In the current situation, decades of progress in reducing poverty and increasing financial inclusion are at risk. Many of these excluded groups, especially the "micro", are excluded from conventional sources of financing in the formal banking system. The past few years have seen tremendous growth in institutions designed to serve this large unbanked segment: charitable and for-profit MFIs, which have around 140 million clients worldwide. However, from the socalled Fintechs, using digital technology to reach the poor and a wide range of other non-bank financial companies, inclusive solutions can be enabled with the use of new technologies (Suarez and Al. (2019).

AI models can help the mapping, management, and realization of predictive models and forecasts that reduce uncertainty and support intelligent decision making. Blockchain enables decentralization, democratization, and digitization. Decentralization to facilitate more efficient asset operations redistributes the market power of intermediaries. Democratization favours peer exchange, increasing market dynamism. Digitization increases traceability, increasing transparency. All of the above opens the way for innovations and collaboration between agencies was not possible before, as a result of the development of extensive margins from increasing efficiency, reducing verification costs, as well as, it could also mean more privacy and security, preventing data leakage.

4.4.3. Political crisis. Social isolation measures put pressure on the credibility of governments when citizens and rival political parties question their adequacy or proportionality, demanding more transparent information to assess the relevance of the measures or their disappearance as well as, these pressures occur both internally between different administrations of the same country or between governments of different countries, in the case of border closures.

Lack of confidence in also is produced at epidemiological data, for example, China has been accused of provides inaccurate information on the development of the disease, crucial information that could have been prevented the pandemic from spreading with so speed to the rest of the world, and the development of policies to prevent or mitigate its expansion and impacts.

The centralization of information and the lack of transparency, both are creating tensions between administrations and citizens and, if they are not managed successfully, they could make the tensions chronic deriving to a political crisis.

On the other hand, the urgent mobilization of all available financial resources to face the effects of the COVID19 outbreak on health, companies, and citizens is necessary, however, it generates significant risks from the accountability point of view.

The necessary accountability not only at the financial audit level, but also for compliance, as well as performance assessment, respecting the criteria of Economy, Efficiency, and Efficiency, communicating and 
reporting in a clear, relevant and timely manner from sufficient, reliable and relevant information, thus necessaries for democracy and avoiding the political crisis.

Let's analyze the potential to solve this crisis of trust through the use of Blockchain from the contact tracking proposals for covid19.

Since the disease is also transmitted by asymptomatic individuals, it is not only necessary to trace positive cases, but to analyze social interactions seems an important tool.

Many automatic contact tracking methods have been suggested using ubiquitous smartphones to facilitate data collection. These methods can easily generate a detailed graph of social interactions, at the same time, doubts arise on the privacy side. Scientific research points to the possibility of considering efficient, and effective COVID19 transition solutions that allow us to respect our fundamental freedom, tracing the history of social interactions of the last 14 days when there is positive taking advantage of Bluetooth (low energy) technology that they enjoy our cell phones.

Bluetooth methods may differ in the controls the individual has over their data. On the one hand, the model proposed by the Italian government "focused on the government", while Google and Apple propose a model "focused on the individual". In Goole-Apple's initial proposal, highly focused on privacy, no encounter record carries one's phone, and all encounters of more than 14 days are deleted forever, only those users who voluntarily decide to share their data. In Italy's proposal, the data collected from a found positive are sent to a government agency that calculates the risk of contagion for each indication encouraging those who have a specific risk of contagion.

The transmission of meeting data is encrypted (being digitally signed), this being a crucial step to prevent data from falling into the wrong hands. However, if the data is made available to the appropriate agency, using zero-knowledge tests and complex cryptography to reconcile privacy and correctness is crucial in both cases, first because of the massive scale involved in a state solution, second because an individually adopted mass voluntary adoption is easier to happen if understood the effectiveness of the proposed solution.

Transparency could be provided by a public blockchain platform, without permission and truly decentralized because it allows the verification and immutability of the data, and therefore establishing the truth, are the key elements of the systems themselves. The blockchain platform is a decentralized database that guarantees 3 properties:

1. Anyone can write an entry in the database.

2. Everyone can read an entry in the database.

3. No one can modify, delete or change the order of the entries in the database.

In neither of the above two solutions is there a common and decentralized global vision of social interactions, only in the case of an Italian proposal can we speak of a common national vision of the number of close centralized meetings on a given day. Although the government could benefit from correct and efficient learning of the current level of social interaction in the country to decide the best way to choose a new policy or adjust an old one, on the other hand, the limitation is that private citizens only have their vision and they cannot determine whether the national vision held by the government is the sum of their individual opinion.

A decentralized common national vision would allow citizens to access the reasons behind the government's decision, a crucial ingredient of democracy. Simply having the government share the information on which it based its own decision with the public is not enough, due to the crisis of confidence generated about the data communicated by the institutions, therefore, the solution to provide credibility would be affected to the public the information you share is, in fact, the truth (Micali, 2020).

\section{Conclusion}

The global pandemic generated by COVID-19 in today's globalized world is having an unprecedented disruptive impact in contemporary history. To the characteristics and severity of the disease, other factors have been added, such as the insufficient structure of the health systems, the lack of coordination and control in the management between governments and administrations, and the low recovery capacity of some productive sectors. 
The harshness of the consequences of isolation, the majority strategy adopted by the states as a short-term solution, has generated an economic shock, both in world demand and supply, and requires forceful massive aid packages for the productive sectors. However, the magnitude, breadth, and speed of stimulus packages from governments and institutions cause the financial needs of the public sector to increase abruptly, a fact that, if not properly managed, can lead to the second great financial crisis of 21 century. At the same time, doubts arise about the reliability and transparency on the part of some institutions, generating a crisis of confidence both internally and between countries, with the consequent risk of a political crisis.

Governments around the world must propose new forms of governance, financial management and control to manage the current deep crisis and be able to anticipate new crises in the future. Emerging technologies like Blockchain, AI, IoT can offer important solutions.

Blockchain enables decentralization, democratization, and digitization. Decentralization to facilitate more efficient asset operations redistributes the market power of intermediaries. Democratization favours the exchange between pairs, increasing the dynamism of the market. Digitization increases traceability, increasing transparency. All of the above paves the way for innovations and collaboration by increasing efficiency, reducing verification costs, as well as improving privacy and security, avoiding data leakage.

AI models can assist in mapping, management, and forecasting and predictive modelling, thereby reducing uncertainty and supporting smart decision making. DL has allowed researchers to increase this complexity to levels that not only appear quantitatively but also qualitatively different from before. DLs increase the range of data that can be managed and increase the ability to analyze data across and outside of corporate data silos by improving the ability of decision-makers to detect opportunities, avoid threats, make better decisions while enabling a more democratized process.

IoT allows the communication of information to people and systems, facilitates control and automation, providing data so that users can understand the process. and analyze opportunities to save costs. Social robotics allows robots to be built and programmed to operate in complex real-world settings.

However, there are limitations to overcome so that they can unleash their full potential, in terms of energy efficiency and implementation difficulties in the case of Blockchain, overcome algorithmic bias, lack of transparency, and significant AI entry barriers, as well as the challenges of security, scalability, interoperability of IoT.

\section{References}

1. ACCA (2019). Audit and Technology. https://www.accaglobal.com/us/en/professionalinsights/technology/audit-and-tech.html.

2. Cheong et al. (2020). Smart Audit: the digital transformation of the audit. ECA Documents. https://www.eca.europa.eu/Lists/ECADocuments/JOURNAL20_01/JOURNAL20_01.pdf.

3. Colombo (2020). Resilient Health Systems: What we are learning from the COVID-19 crisis. https://www.oecd-forum.org/users/382555-francesca-colombo/posts/64973-resilient-health-systemswhat-we-are-learning-from-the-covid-19-crisis.

4. CPA Canada (2018). Audit Considerations Related to Cryptocurrency Assets and Transactions. https://www.cpacanada.ca/en/business-and-accounting-resources/audit-and-assurance/canadianauditingstandards-cas/publications/cryptocurrency-audit-considerations.

5. Dai and Vasarhelyi (2017). Toward blockchain-based accounting and assurance. Journal of Information Systems, 31(3), 5-21. American Accounting Association. DOI: 10.2308/isys-51804.

6. Escola and Kadi (2017). Strategic Thinking for Public Management: Confluence for the PLanning, Organization, Direction and http://ojs.urbe.edu/index.php/coeptum/article/download/1677/3804?inline=1.

7. EU Blockchain Observatory and Forum (2020). Convergence of Blockchain, AI and IoT. https://www.eublockchainforum.eu/sites/default/files/report_convergence_v1.0.pdf.

8. Friga and Paul (2020). Scenario Planning for Coronavirus. Inside Higher Ed. https://www.insidehighered.com/views/2020/03/13/using-strategic-thinking-and-scenario-planningdeal-coronavirus-opinion.

9. Lopez (2013). An Introduction on the Internet of Things (IoT). https://www.cisco.com/c/dam/en_us/solutions/trends/iot/introduction_to_IoT_november.pdf. 
10. Marr (2018). What is Industry 4.0? Here's A Super Easy Explanation For Anyone. Forbes. https://www.forbes.com/sites/bernardmarr/2018/09/02/what-is-industry-4-0-heres-a-super-easyexplanation-for-anyone/\#5a10001f9788.

11. Mawela (2012). Strategic planning for transformational government: A South African perspective. Eleadership Conference on Sustainable e-Government and e-Business Innovations (E-LEADERSHIP), Pretoria, 2012, pp. 1-9, DOI: 10.1109/e-Leadership.2012.6524705.

12. Micali and Silvio (2020). Algorand's Approach to COVID-19 Tracing. https://www.algorand.com/resources/blog/silvio-micali-approach-to-covid-19.

13. Narula et al. (2018). ZkLedger: Privacy-Preserving Auditing for Distributed Ledgers. https://www.usenix.org/system/files/conference/nsdi18/nsdi18-narula.pdf.

14. Regalado (2020). What is group immunity and how it can stop the coronavirus. https://www.technologyreview.es/s/12035/que-es-la-inmunidad-de-grupo-y-como-puede-detener-alcoronavirus.

15. Rozario and Thomas (2019). Reengineering the audit with blockchain and smart contracts. Journal of Emerging Technologies in Accounting, 16(1), 21-35. https://doi.org/10.2308/jeta-52432.

16. Rozario and Vasarhelyi (2018). Auditing with Smart Contracts. International Journal of Digital Accounting Research 18. chromeextension://ohfgljdgelakfkefopgklcohadegdpjf/https://pdfs.semanticscholar.org/3122/b35e03fb $\underline{758108}$ 550327d493e2e4748ad2d.pdf.

17. Scarpetta and Steffano (2020). Beyond Containment: Health systems responses to COVID-19 in the $O E C D$. Available at: $\quad$ https://read.oecd-ilibrary.org/view/?ref=119_119689ud5comtf84\&title=Beyond_Containment:Health_systems_responses_to_COVID-19_in_the_OECD.

18. Setti et al. (2020). Report on the effect of air pollution and the spread of virus in the population. Available at: http://www.simaonlus.it/wpsima/wp-content/uploads/2020/03/COVID19_Position-Paper_Relazionecirca-1\%E2\% 80\%99effetto-dell\%E2\% 80\%99inquinamento-da-particolato-atmosferico-e-la-diffusionedi-virus-nella-popolazione.pdf.

19. Belen Suarez Lopez, David Issó García, Antonio Vargas Alcaide (2019). Blockchain Technology Facing Socioeconomic Challenges. Promise versus Probability. SocioEconomic Challenges, 3(4), 13-24. http://doi.org/10.21272/sec.3(4).13-24.2019. 